\title{
PROFILAXIA PARA TROMBOSE VENOSA PROFUNDA EM PACIENTES COM FRATURAS DE MEMBRO INFERIOR INTERNADOS EM UM HOSPITAL REFERÊNCIA DE GOIÂNIA
}

\author{
PROPHYLAXIS FOR DEEP VENOUS TROMBOSIS IN PATIENTS WITH LOWER \\ LIMB FRACTURES IN A REFERENCE HOSPITAL IN GOIANIA
}

\author{
Lays Rodrigues Santos', Giulliano Gardenghi ${ }^{2}$, Adroaldo Jose Casa Junior ${ }^{3}$
}

\begin{abstract}
Autor para correspondência: Giulliano Gardenghi - ggardenghi@encore.com.br 'Fisioterapeuta. Pós-graduanda em Fisioterapia Traumato-Ortopédica. Fisioterapeuta na empresa Lifecare, prestadora de serviço junto ao Hospital de Urgências de Goiânia, Goiânia, Goiás, Brasil. ${ }^{2}$ Fisioterapeuta. Doutor em Ciências, Coordenador Científico do Hospital ENCORE, Goiás; Coordenador Científico do CEAFI Pós-Graduação, Goiás, Coordenador do Serviço de Fisioterapia do Hospital de Urgências de Goiânia/Lifecare, Coordenador do Serviço de Fisioterapia da UTI do IGOPE (Instituto Goiano de Pediatria) e Coordenador do Curso de Pós-Graduação em Fisioterapia Hospitalar do Hospital e Maternidade São Cristóvão, São Paulo (SP), Brasil. http://orcid.org/0000-0002-8763-561X

${ }^{3}$ Fisioterapeuta. Doutorando em Ciencias da Saúde pela Universidade Federal de Goiás. Especialista em Fisioterapia Traumato-Ortopédica pela Universidade Castelo Branco - RJ. Mestre em Ciências da Saude pela Universidade de Brasilia. Professor da pós-graduação de TraumatoOrtopedia da Mundo Fisio e professor de graduação de fisioterapia da PUC - GOIÁS.
\end{abstract}

RESUMO | Introdução: A trombose venosa profunda (TVP) é caracterizada pela formação de trombos nas veias profundas. É a principal causa de embolia pulmonar (EP), sendo de extrema importância que se realize a profilaxia da TVP. Objetivos: Verificar se a profilaxia para TVP em pacientes com fraturas de membro inferior em um hospital público voltado para atendimento de urgências está sendo eficiente, identificar as categorias de risco para TVP de acordo com a Sociedade Brasileira de Angiologia e Cirurgia Vascular (SBACV) e verificar o número de atendimentos de fisioterapia realizados nessa população durante internação hospitalar. Metodologia: Estudo transversal quantitativo e analítico de 79 pacientes internados com uma ou múltiplas fraturas em membro inferior, entre abril e junho de 2016. Os dados foram coletados nos prontuários, sendo aplicado o protocolo de estratificação de risco para TVP da SBACV. Resultados: Homens corresponderam $75,9 \%$ da amostra. A idade foi de $38,6 \pm 18,2$ anos. $1,3 \%$ da amostra foram estratificados em baixo risco, $74,7 \%$ em médio risco e $24,1 \%$ em alto risco para TVP. $3,8 \%$ da amostra não receberam profilaxia medicamentosa. Os incluídos no estudo receberam atendimento fisioterapêutico a cada 2,6 dias durante período de internação e 2,5\% da amostra apresentaram complicações, sendo um óbito e uma TVP com evolução para TEP. Conclusões: A profilaxia medicamentosa para TVP associado às manobras fisioterapêuticas foram eficazes na maioria dos pacientes estudados. A maior parte dos pacientes foi classificada como em médio risco para desenvolvimento de TVP. $O$ número de sessões de fisioterapia durante o período de internação prestado foi considerado baixo, pela falta de pessoal associada ao serviço público em questão.

Palavras-chave: trombose venosa profunda, fisioterapia, embolia pulmonar.

\begin{abstract}
Introduction: Deep vein thrombosis (DVT) is characterized by the formation of deep vein thrombi. It is the main cause of pulmonary embolism (PE), and prophylaxis of DVT is extremely important. Objectives: To verify if the prophylaxis for DVT in patients with lower limb fractures in a public hospital for urgent care is being efficient, to identify the risk categories for DVT according to the Brazilian Society of Angiology and Vascular Surgery (SBACV) and To verify the number of physiotherapy visits performed in this population during hospitalization. Methodology: A quantitative and analytical cross-sectional study of 79 patients hospitalized with one or multiple fractures in the lower limb between April and June 2016. Data were collected in the medical records, and the protocol of risk stratification for SBPVV was applied. Results: Men corresponded to $75.9 \%$ of the sample. The age was 38.6 \pm 18.2 years. $1.3 \%$ of the sample were stratified at low risk, $74.7 \%$ at medium risk and $24.1 \%$ at high risk for DVT. $3.8 \%$ of the sample did not receive medication prophylaxis. Those included in the study received physiotherapeutic care every 2.6 days during the hospitalization period and $2.5 \%$ of the sample presented complications, one of which was a death and a DVT with evolution to PE. Conclusions: The prophylaxis for DVT associated with physiotherapeutic maneuvers was effective in the majority of patients studied. Most patients were classified as at medium risk for developing DVT. The number of physical therapy sessions during the hospitalization period was considered low due to the lack of personnel associated with the public service in question.
\end{abstract}

Key words: venous thrombosis, Physical Therapy Specialty, pulmonary embolism. 


\section{INTRODUÇÃO}

O desenvolvimento do trombo venoso depende da tríade de Virchow, descrita em 1856, que considera as alterações do fluxo sanguíneo, da crase sanguínea e da parede vascular como responsáveis pelo processo trombótico'. O tromboembolismo venoso (TEV) agrega diversos quadros clínicos, entre eles destacam-se a trombose venosa profunda (TVP), a embolia pulmonar (EP) e a trombose associada a um cateter venoso central ${ }^{2}$. A TVP é uma doença caracterizada pela formação de trombos no sistema venoso profundo, sendo os membros inferiores os mais acometidos ${ }^{3}$. Em sua maioria, os coágulos são pequenos e clinicamente insignificantes, sendo que em alguns casos haverá resolução espontânea sem efeitos adversos. Porém, há uma importante associação entre TVP e EP, e também entre trombos não oclusivos silentes e EP sintomática ou fatal ${ }^{4,5}$.

O quadro clínico local depende, em grande parte, da extensão da área acometida e das veias atingidas, podendo acompanhar-se de manifestações sistêmicas e complicações como a EP6 . De maneira geral os pacientes podem apresentar: dor, desconforto ou sensação de peso em membros inferiores, veias varicosas, edema, hiperpigmentação, eczema de estase, celulite/erisipela, lipodermatoesclerose e úlceras de estase 7 .

Por ser a principal causa de EP, é de extrema importância que se realize a profilaxia da TVP, pois a embolia pulmonar pode ser a primeira manifestação clínica da doença e costuma ser fatal em $0,2 \%$ dos pacientes internados ${ }^{8}$.

No Brasil, algumas entidades, como ○ Conselho Federal de Medicina, a Associação Médica Brasileira, o Ministério da Saúde e a Sociedade Brasileira de Angiologia e Cirurgia Vascular (SBACV), propuseram diretrizes de combate a TVP, dando ênfase a ações especificas sobre cada um dos fatores de risco associados. De acordo com a diretriz de TVP são considerados pacientes de baixo risco aqueles que fizeram operação com idade menor de 40 anos sem outros fatores de risco, operações menores em pacientes de mais de 40 anos sem outro risco que não idade ou trauma menor. Os pacientes estratificados em risco moderado são aqueles que sofreram trauma menor, cirurgia maior em pacientes de 40 a 60 anos sem fatores adicionais de risco ou cirurgia em pacientes com menos de 40 anos tomando estrógenos. Já os pacientes considerados de alto risco são aqueles que passaram por cirurgia geral com idade maior de 60 anos, cirurgia geral em pacientes de 40-60 anos com fatores de risco adicionais, cirurgia maior em pacientes com história de TVP ou EP pregressa ou trombofilia, grandes amputações, cirurgias ortopédicas maiores, cirurgias maiores em pacientes com neoplasias, cirurgias maiores em pacientes com outros estados de hipercoagulabilidade ou traumas múltiplos associados fraturas de pelve, quadril ou membros inferiores ${ }^{6}$.

A fisioterapia motora é recomendada para todos os pacientes com diferentes riscos de TVP, atuando como adjuvante à terapia farmacológica e ainda nos casos com contraindicação ao uso de anticoagulantes ${ }^{9,10}$. De acordo com a natureza das afecções e doenças ortopédicas, como traumas, artroplastias, principalmente em quadril e joelho, predispõe-se a ocorrência de TVP. O posicionamento do membro acometido durante a intervenção, - edema localizado e as limitações quanto à mobilidade no pós-operatório imediato, têm participação na estase venosa com a consequente redução do fluxo sanguíneo ${ }^{1}$.

Além da terapia medicamentosa e da fisioterapia existem ainda métodos mecânicos para profilaxia da TVP, as meias de compressão graduada, a compressão pneumática intermitente (CPI) e - massageador venoso plantar. Tais métodos aumentam a velocidade de fluxo sanguíneo e reduzem a estase venosa. Apesar dos benefícios de não apresentarem risco para sangramento, estes métodos podem ser de difícil instalação ou manutenção pela limitação à movimentação do paciente. Além disso, existe contraindicação em fraturas expostas, infecção de membros inferiores, insuficiência cardíaca grave e insuficiência arterial periférica $^{12}$. Em contrapartida, dados do American College of Chest Physicians sugerem a utilização dos métodos mecânicos em adição à profilaxia farmacológica durante a internação em pacientes com alto risco para TVP em cirurgias ortopédicas maiores $^{13}$. Com base nesse contexto, 0 enfoque 
desse estudo é justificável uma vez que seus resultados poderão contribuir positivamente para a redução dos casos de TVP no ambiente hospitalar em pacientes com fraturas de membros inferiores, diminuindo também 0 tempo de internação desses pacientes e consequentemente provocando redução de gastos hospitalares, além de fornecer subsídios para posteriores trabalhos que visem análise profunda sobre o tema.

Esse estudo tem por objetivos verificar se a profilaxia utilizada para TVP em pacientes com fraturas de membro inferior em um hospital público voltado para atendimento de urgências no Estado de Goiás está sendo eficiente; Identificar as categorias de risco para TVP de acordo com a SBACV nos pacientes com fraturas de membro inferior internados; Verificar o número de atendimentos de fisioterapia realizados nos pacientes com fraturas de membro inferior internados em risco para TVP.

\section{MÉTODOS}

Trata-se de um estudo transversal, de caráter quantitativo e analítico de 79 pacientes internados no Hospital de Urgências de Goiânia (HUGO), com diagnóstico de uma ou múltiplas fraturas em membro inferior, nos meses de abril a junho de 2016. A seleção destes foi realizada por meio da utilização dos seguintes critérios de inclusão: ter idade acima de 18 anos, homens e mulheres com diagnóstico clínico de uma ou múltiplas fraturas de membros inferiores internados no hospital nos meses de abril a junho de 2016. Foram excluídos os pacientes portadores de doenças neurológicas e coagulopatias, pacientes em tratamento ambulatorial (internação menor que 24 horas) e obstétricos.

Após aprovação do Comitê de Ética em Pesquisa do HUGO sob número de parecer 1.517.073, os dados foram coletados nos prontuários impressos e eletrônicos dos pacientes, além de ter sido realizada a estratificação dos riscos para TVP com aplicação do protocolo específico da SBACV, conforme descrito na figura 01 .

Para a estratificação de risco para TVP de cada paciente, foram pesquisados fatores clínicos, medicamentosos e cirúrgicos, segundo o protocolo preconizado pela $\mathrm{SBACV}^{6}$. A correta utilização da profilaxia foi analisada segundo critérios da SBACV, que estabelece classificação de riscos (alto, médio e baixo) através de pontuações. Para concluir o preenchimento do protocolo, o paciente foi entrevistado para maior fidedignidade da estratificação de risco.

A tabulação e a análise estatística descritiva foram realizadas no Microsoft Excel, versão 2003. As variáveis quantitativas serão a seguir apresentadas em médias, desvio-padrão, mínimas, máximas, mediana e intervalo interquartil. 


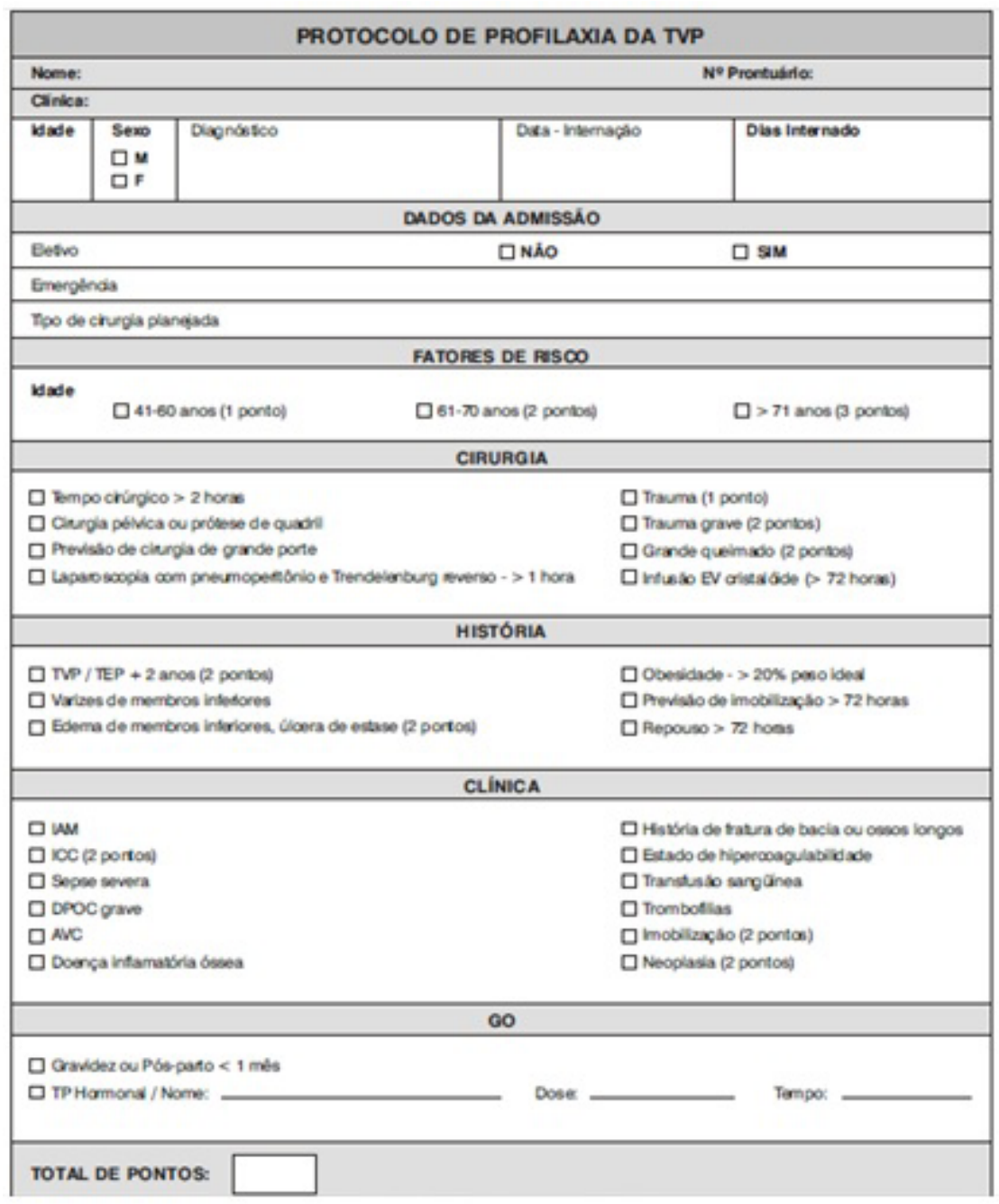

Figura 01. Questionário recomendado pela SBACV para investigação do risco e profilaxia da TVP

\section{RESULTADOS}

Foram analisados 79 pacientes. A média de idade foi $38,6 \pm 18,2$ anos. Homens corresponderam a $75,9 \%$ da amostra. A média de dias de internação foi de 22,9 (mediana 18, intervalo interquartil 16). No que tange a estratificação de risco para TVP, $1,3 \%$ apresentaram baixo risco, $74,7 \%$ médio risco e $24,1 \%$ alto risco.

Dos 79 pacientes, três $(3,8 \%)$ não receberam profilaxia medicamentosa para TVP, sendo um estratificado em baixo risco e dois em médio risco. Todos os pacientes receberam atendimento fisioterapêutico. A medicação utilizada foi heparina de baixo peso molecular (enoxaparina sódica $40 \mathrm{mg}$ ), uma vez ao dia. Houve um óbito por TEP e um episódio de TVP com evolução para TEP, correspondendo a $2,5 \%$ da amostra. Ambos os pacientes foram estratificados em médio risco e receberam, além da profilaxia medicamentosa, atendimento fisioterapêutico. A média de atendimento fisioterapêutico recebido durante internação foi de 8,8 atendimentos, com mediana de 6 e intervalo interquartil de 8 . $O$ tratamento fisioterapêutico mais prevalente foi baseado na associação de movimentação ativa dos membros inferiores com exercícios respiratórios e, quando possível, deambulação precoce, além de orientações gerais, tomando por base as indicações para profilaxia relacionadas a exercício recomendadas pela SBACV (figura 02). Nenhum paciente utilizou métodos mecânicos para prevenção de TVP, ou seja, meias de compressão e compressão pneumática 
intermitente ${ }^{6}$. O resumo dos resultados está representado na tabela 01.

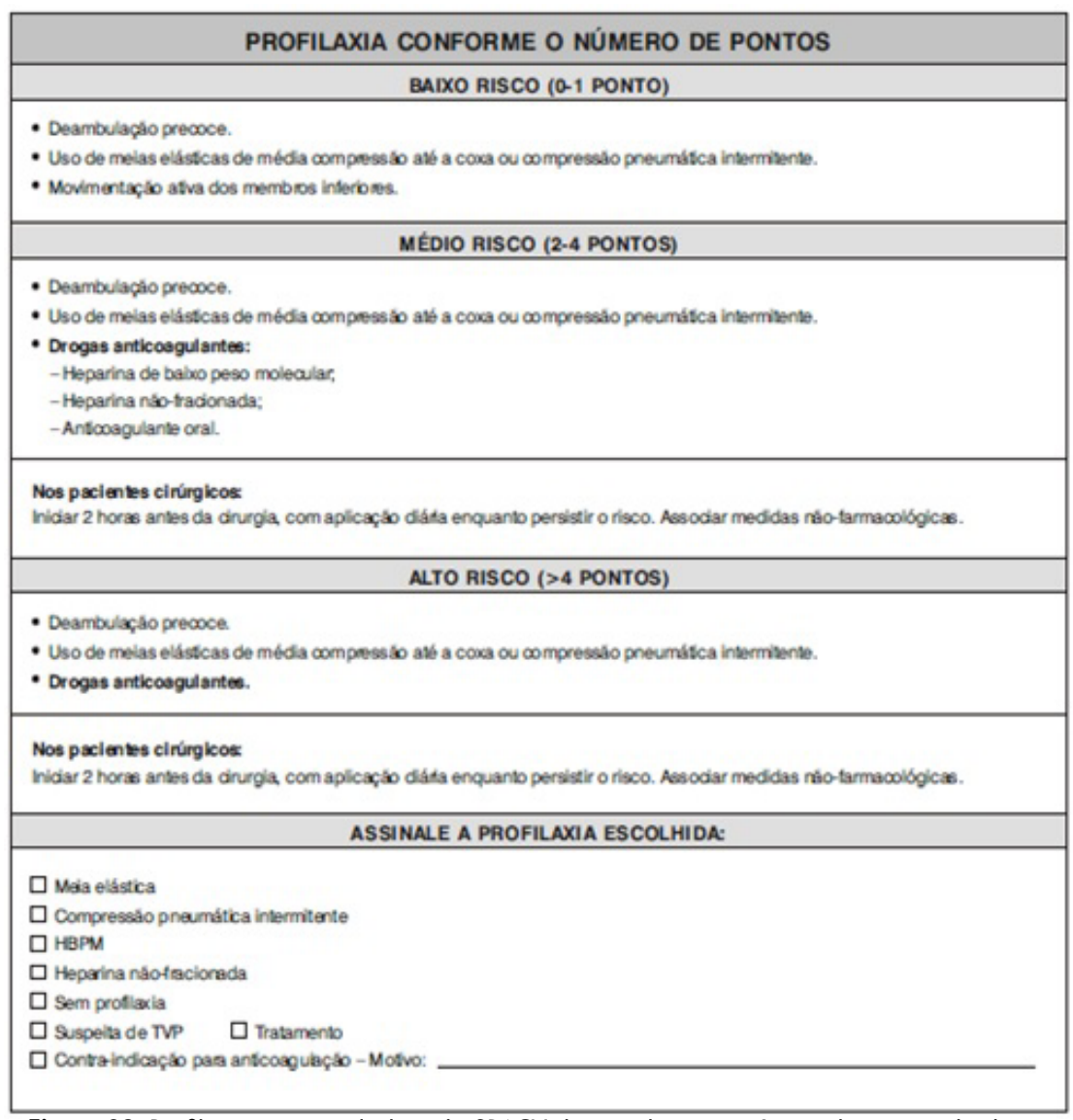

Figura 02. Profilaxia recomendada pela SBACV de acordo com o número de pontos obtidos no questionário de investigação de risco para TVP

Tabela 01. Nível de risco para TVP, número de TVP, TEP e óbito, profilaxia medicamentosa e fisioterapêutica

\begin{tabular}{cccccc}
\hline $\begin{array}{l}\text { Nível de } \\
\text { Risco }\end{array}$ & $\mathrm{n}(\%)$ & $\begin{array}{l}\text { TVP/TEP } \\
\text { (episódios) }\end{array}$ & Óbito(\%) & $\begin{array}{l}\text { Receberam profilaxia } \\
\text { medicamentosa (\%) }\end{array}$ & $\begin{array}{l}\text { Fisioterapia } \\
(\%)\end{array}$ \\
\hline & & & & & 100 \\
Baixo & 1,3 & 0 & 0 & 100 & 100 \\
Médio & 74,7 & 2,5 & 1,3 & 72,15 & 100 \\
Alto & 24,1 & 0 & 0 & 100 & 100 \\
\hline
\end{tabular}


A tabela abaixo (tabela 02) mostra os tipos de fraturas com que os pacientes foram diagnosticados.

Tabela 02. Tipos de fraturas encontrados

\begin{tabular}{lcc}
\hline \multicolumn{1}{c}{ Tipo de Fratura } & $\mathrm{n}$ & $\%$ \\
\hline Membro Inferior Esquerdo & 1 & 1,3 \\
Quadril & 1 & 1,3 \\
Fêmur & 33 & 41,7 \\
Fêmur e perna & 3 & 3,8 \\
Fêmur e perna bilateral & 1 & 1,3 \\
Joelho & 1 & 1,3 \\
Patela & 1 & 1,3 \\
Perna & 9 & 11,3 \\
Perna bilateral & 1 & 1,3 \\
Perna e tornozelo & 1 & 1,3 \\
Tíbia & 8 & 10,1 \\
Tíbia e antepé & 1 & 1,3 \\
Platô tibial & 6 & 7,5 \\
Planalto tibial & 1 & 1,3 \\
Bimaleolar & 1 & 1,3 \\
Tornozelo & 9 & 11,3 \\
Metatarsos & 1 & 1,3 \\
Total & & 100 \\
\hline & 79 & \\
\hline
\end{tabular}


Os principais fatores de risco encontrados foram a idade maior de 40 anos e tempo de imobilização maior que 72 horas, demonstrados na tabela abaixo (tabela 03 ).

Tabela 03. Principais fatores de risco para TEV encontrados

\begin{tabular}{ll}
\hline \multicolumn{1}{c}{ Fator de risco } & $\mathrm{n}(\%)$ \\
\hline Idade acima de 40 anos & 37,8 \\
Tempo de imobilização & 98,7 \\
maior que 72 horas & \\
\hline
\end{tabular}

\section{DISCUSSÃO}

A incidência mundial de EP por complicação de TVP é de 50/100.000 habitantes por ano, sendo diretamente proporcional ao aumento da idade. No Brasil é relatado $0,6 / 1.000$ habitantes no ano ${ }^{14}$. Há estimativa de que $25-50 \%$ dos pacientes com TVP desenvolverão a síndrome pós-trombótica com redução da qualidade de vida ${ }^{10,15}$.

A prevenção da TVP é fundamental e necessária para se evitar suas complicações agudas, como o TEP, ou crônicas, como a síndrome pós-trombótica. As medidas profiláticas são cada vez mais discutidas e indicadas, juntamente com $\circ$ aprimoramento dos protocolos.

Os fatores de risco a serem considerados para diagnóstico da TVP são: idade, trombofilia, varizes, obesidade, infecção, trauma, gravidez e puerpério, anestesia com duração maior que 30 minutos, anestesia geral, uso de estrógenos, insuficiência cardíaca, acidente vas-cular cerebral, doença respiratória grave, doença inflamatória intestinal, infarto do miocárdio, insuficiência arterial, quimioterapia, síndrome nefrótica, cateteres centrais, história prévia de TVP e/ou embolia pulmonar, câncer, paralisia, paresia ou imobilização recente do membro inferior, recente confinamento no leito por mais que três dias ou uma grande cirurgia dentro de quatro semanas, sensação dolorosa localizada ao longo da distribuição do sistema venoso profundo, edema em todo o membro inferior, edema na panturrilha, edema depressível e dilatação das

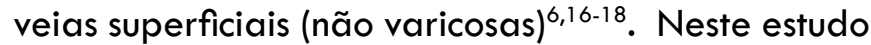
os principais fatores de risco encontrados foram trauma, idade maior que 40 anos e imobilização do membro inferior.

A importância e os benefícios de uma correta e efetiva profilaxia medicamentosa estão bem documentados, sendo amplamente defendidos por reduzir as ocorrências de TVP e TEP. Neste estudo, $96,2 \%$ dos pacientes receberam profilaxia medicamentosa. Busato e cols. realizaram um estudo de coorte na Santa Casa de Misericórdia de Ponta Grossa para verificar a utilização da profilaxia para TVP em pacientes cirúrgicos e clínicos e destes, apenas $53,85 \%$ receberam a profilaxia medicamentosa corretamente ${ }^{19}$. No estudo de Garcia e cols., de 182 pacientes que deveriam receber profilaxia medicamentosa para TVP, apenas 56 receberam a medicação ${ }^{20}$. Em outro estudo prospectivo, cujo objetivo foi verificar se a profilaxia medicamentosa estava sendo realizada de maneira rotineira e correta no Hospital Geral de Roraima, $100 \%$ dos pacientes da ortopedia não receberam profilaxia medicamentosa para TVP ${ }^{14}$.

No estudo de Engelhorn e cols., os ortopedistas utilizaram a profilaxia medicamentosa em apenas quatro pacientes num total de 2421 . Em pacientes ortopédicos, as taxas de TVP, na ausência de profilaxia, são de 40 a $60 \%$ e de $2-5 \%$ sob regime de profilaxia ${ }^{22}$. Pode-se verificar que mesmo com a disponibilidade de vários esquemas profiláticos 
tecnicamente adequados, farmacológicos e/ou fisioterapêuticos, nem sempre as equipes assistenciais optam pela adoção de tais recursos.

Todos os pacientes analisados no estudo presente receberam atendimento fisioterapêutico regularmente. Foram realizadas mobilizações ativas e passivas para membros inferiores associados a exercícios respiratórios, deambulação precoce e orientações gerais. No ENDORSE, um estudo multicêntrico realizado em diversos países, apenas metade dos 68.183 pacientes receberam os métodos profiláticos recomendados, incluindo a fisioterapia ${ }^{23}$. Campos, Albuquerque e Braga em sua pesquisa, concluíram que a terapia física é um fator importante na prevenção do TEV. Eles realizaram um estudo piloto com uma amostra de 30 indivíduos e demonstraram que os exercícios de bomba sural (flexão plantar do tornozelo), realizado de forma ativa com o paciente no leito, diminui a estase venosa comparada à forma passiva, e que o fortalecimento da musculatura da panturrilha é capaz de melhorar a hemodinâmica venosa deste segmento ${ }^{24}$. Para Cunha e Veado, a mobilização precoce deve ser incentivada nas primeiras 48 horas do pósoperatório, sendo vital para prevenir complicações como broncopneumonia, infecção urinária, TEV e também para ajudar a recuperar a confiança e motivação do paciente. Além disso, medidas físicas, como elevação dos membros inferiores, fisioterapia e meias elásticas de compressão graduada, são amplamente aplicáveis e de baixo custo, melhorando o fluxo sanguíneo venoso femoral e diminuindo a incidência de TVP $^{25}$, motivo pelo qual deveriam ser adotadas em todo paciente admitido em ala ortopédica com fraturas de membros inferiores, desde que não ocorra contraindicação médica para tais procedimentos.

Nosso estudo tem limitações que devem ser apontadas. Não houve um grupo controle que não fizesse fisioterapia, para que pudéssemos realizar uma análise comparativa do real efeito da mesma. Por questões de falta de pessoal especializado (fisioterapeutas), não foi realizada a fisioterapia diariamente, o que poderia influenciar positivamente em uma menor incidência de TVP ou EP, que embora baixa em nosso estudo, quando comparada a outros centros descritos nesse artigo, ainda representou aos indivíduos acometidos e à equipe assistencial, um desfecho extremamente significante e negativo.

\section{CONCLUSÕES}

Os resultados deste estudo demonstraram que a profilaxia medicamentosa está sendo realizada de forma efetiva em pacientes com fraturas de membro inferior em um hospital público voltado à assistência de urgências no Estado de Goiás. Apesar de não haver um grupo controle que não realizasse fisioterapia para podermos analisar $\circ$ efeito da mesma nestes pacientes, todos os inclusos no estudo receberam atendimento fisioterapêutico a cada 2,6 dias e 2,5\% da amostra apresentaram complicações. Além disso, concluímos que apesar de sua eficácia já ter sido comprovada em diversos estudos, a profilaxia mecânica para prevenção da TVP ainda não é utilizada no hospital em questão.

\section{CONTRIBUIÇÕES DOS AUTORES}

Santos LR foi responsável pela idealização do projeto de pesquisa, coleta de dados, redação do manuscrito e revisão final do manuscrito. Gardenghi $G$ foi responsável pela idealização do projeto de pesquisa, redação do manuscrito, análise dos dados e revisão do manuscrito. Casa Junior, AJ, foi responsável pela idealização do projeto de pesquisa.

\section{CONFLITOS DE INTERESSES}

Nenhum conflito financeiro, legal ou político envolvendo terceiros (governo, empresas e fundações privadas, etc.) foi declarado para nenhum aspecto do trabalho submetido (incluindo mas não limitandose a subvenções e financiamentos, conselho consultivo, desenho de estudo, preparação de manuscrito, análise estatística, etc).

\section{REFERÊNCIAS}

1. Maffei FHA, Rollo HA. Trombose venosa profunda dos membros inferiores: incidência, patologia, patogenia, fisiopatologia e diagnóstico. In: Maffei FHA, Lastoria S, Yoshida WB, Rollo HA. Doenças Vasculares Periféricas. Rio de Janeiro: Medsi; 2002. P.1363-86

2. Ribeiro MA, Netto PG, Lage SG. Desafios na profilaxia do tromboembolismo venoso: abordagem do paciente crítico. Rev Bras Ter Int. 2006; 18(3):316-19

3. Jacques NMP, Francisco-Jr J, Burihan E. Trombose Venosa Profunda. In: Burihan E, Ramos RR. Condutas em Cirurgia. São Paulo: Atheneu; 2001. P.701-20

4. Lieberman JR, Huo MM, Hanway J, Salvati EA, Sculco TP, 
Sharrock NE. The prevalence of deep venous thrombosis after total hip arthroplasty with hypotensive epidural anesthesia. J Bone Joint Surg Am. 1994;76(3):341-8

5. Pellegrini VD Jr, Clement D, Lush-Elman C, Keller GS, Evarts $C M$. The natural history of thromboembolic disease following hospital dis- charge after total hip arthroplasty: the case for routine surveillance. Clin Orthop Relat Res. 1996;(333):27-40

6. Maffei FHA, Caiafa JS, Ramacciotti E, Castro AA para o Grupo de Elaboração de Normas de Orientação Clínica em Trombose Venosa Profunda da SBACV. Normas de orientação clínica para prevenção, diagnóstico e tratamento da trombose venosa profunda (revisão 2005). Salvador: SBACV [Internet]. 2005 [acesso em 20 jul 2016]. Disponível em: http://www.jvascbr.com.br/Arquivo_4.pdf

7. Sociedade Brasileira de Angiologia e Cirurgia Vascular. Terapia de compressão de membros inferiores. São Paulo: Associação Médica Brasileira, Conselho Federal de Medicina; 2011

8. Kearon C. Natural history of venous thromboembolism. Circulation. 2003;107(23suppl1):122-130. doi: 10.1161/01. CIR.0000078464.82671.78

9. Rocha AT, Paiva EF, Lichtenstein A et al. Tromboembolismo Venoso: Profilaxia em Pacientes Clínicos. Rev Assoc Med Bras. 2009;55(2):95-107

10. Pitta GBB, Leite TL, Silva MDC, Melo CFL, Calheiros GA. Evaluation of the use of prophylaxis for deep venous thrombosis in a teaching hospital. J Vasc Bras. 2007;6(4):34451. doi: 10.1590/S1677-54492007000400008

11. Menzin J, Richner R, Huse D, Colditz GA, Oster G. Prevention of deep-vein thrombosis following total hip replacement surgery with enoxaparin versus unfractionated heparin: a pharmacoeconomic evaluation. Ann Pharmacother. $1994 ; 28(2): 271-5$

12. Geerts WH, Pineo GF, Heit JA, Berqquist D, Lassen MR, Colwell CW et al. Prevention of Venous Thromboembolism. The Seventh ACCP Conference on Antithrombotic and Thrombolytic Therapy. Chest. 2004; 1 26:338S-400S. doi: 10.1378/chest.126.3_suppl.338S

13. Falck-Ytter Y, Francis CW, Johanson NA, Curley C, Dahl OE, Schulman S et al. Prevention of VTE in orthopedic surgery patients: Antithrombotic Therapy and Prevention of Thrombosis, 9th ed: American College of Chest Physicians Evidence Based Clinical Practice Guidelines. Chest. 2012 ; 141 (2Suppl):e278S-325S. doi: 10.1378 /chest. 1 1-2404

14. Pereira CA, Brito SS, Martins AS, Almeida CM. Deep venous thrombosis prophylaxis: practical application and theoretical knowledge in a general hospital. J Vasc Bras. 2008;7(1):18-27. doi: 10.1590/S167754492008000100005

15. Penha GS, Damiano AP, Carvalho T, Lain V, Serafim JD.
Mobilização precoce na fase aguda da trombose venosa profunda de membros inferiores. J Vasc. Bras 2009;8(1):7785

16. Wells PS, Hirsh J, Anderson DR, Lensing AW, Foster G, Kearon $C$ et al. Accuracy of clinical assessment of deep-vein thrombosis. Lancet. 1995; 345(8961):1326-30

17. Wells PS, Anderson DR, Bormanis J, Guy F, Mitchell M, Gray $L$ et al. Value of assessment of pretest probability of deep-vein thrombosis in clinical management. Lancet. 1997;350(9094):1795-8. doi: 10.1016/S01406736(97)08140-3

18. Anand SS, Wells PS, Hunt D, Brill-Edwards P, Cook D, Ginsberg JS. Does this patient have deep vein thrombosis? JAMA. 1998;279(14):1094-9. [Published errata appear in JAMA 1998 May 27;279(20):1614 and 1998 Jul 22$29 ; 280(4): 328]$

19. Busato CR, Gomes RZ, Costa DMM, Zubiolo TFM. Avaliação da tromboprofilaxia em hospital geral de médio porte. J Vas Bras. 2014;13(1):5-1 1. doi: 10.1590/ jvb.2014.003

20. Garcia ACF, Sousa BV, Volpato DE, Deboni LM, Souza $M V$, Martinelli $R$ et al. Realidade do uso da profilaxia para trombose venosa profunda: da teoria à prática. J Vasc Bras. $2005 ; 4(1): 35-41$

21. Engelhorn ALV, Garcia ACF, Cassou MF, Birckholz L, Engelhorn CA. Profilaxia da trombose venosa profunda: estudo epidemiológico em um hospital escola. J Vasc Bras. 2002; 1 (2):97-102

22. White RH, Romano PS, Zhou H. A population-based comparison of the 3-month incidence of thromboembolism after major elective/urgent surgeries [abstract] Thromb Haemost. $2001 ; 86: 2255$

23. Goubran HA, Sholkamy S, El-Haddad A, Mahmoud A, Rizkallah MA, Sobhy G. Venous thromboembolism risk and prophylaxis in the acute hospital care setting: report from the ENDORSE study in Egypt. Thrombosis Journal. 2012; 10:20. doi: $10.1186 / 1477-9560-10-20$

24. Campos CCC, Albuquerque PC, Braga IJS. Avaliação do fluxo venoso da bomba sural por ultrassonografia doppler durante cinesioterapia ativa e passiva: um estudo piloto. J Vasc Bras. 2008;7(4):325-32. doi: 10.1590/S167754492008000400007

25. Cunha U, Veado MAC. Fratura da extremidade proximal do fêmur em idosos: independência funcional e mortalidade em um ano. Rev Bras Ortop. 2006; 41 (6): 195-99 